\title{
Mind the gap: Improving discharge communication between secondary and primary care
}

Aynsley Cresswell, Matthew Hart, Ondrej Suchanek, Tania Young, Laurence Leaver, Stephen Hibbs Oxford University Hospitals NHS Trust, UK

\begin{abstract}
Foundation year doctors (FYDs) write most hospital discharge communication, although they have minimal training in this skill. Poor quality discharge summaries increase the risk of adverse events and rehospitalisation. With a multidisciplinary team approach, we developed a list of "golden rules" for good discharge communication. Against these standards, we analysed the quality of electronic inpatient discharge documentation (eIDD) sent over two months from OUH Trust. We found one third of elDDs were missing details of the discharging doctor. In $68 \%$, changes to medications were not documented clearly and follow-up was not completed in $40 \%$.
\end{abstract}

To improve this suboptimal state, we implemented interactive teaching sessions for FYDs, designed an e-learning module, and suggested software changes to the current electronic discharge proforma. Early re-audit one month after the first teaching sessions did not demonstrate any significant improvement. However, re-auditing after twelve months is planned.

Through data collection and discussion with key stakeholders, we have identified standards for discharge communication. We developed interventions to help the trust achieve these standards, aiming to enhance patient safety in the peri-discharge period. While discharge communication is delegated to less-experienced team members, they should receive clear guidance and training.

\section{Problem}

Discharge summaries are a standard communication tool delivering important clinical information from inpatient to ambulatory care. Foundation year doctors (FYDs) write the majority of electronic inpatient discharge documentation (elDDs), yet they receive minimal training in how to do so. This increases the risk of poorquality eIDDs being produced. A quality improvement project was conducted (in conjunction with a "Management in Medicine" Workshop, based at Green Templeton College, Oxford) aiming to improve the content of elDDs for inpatients within the Oxford University Hospitals (OUH) NHS trust.

From an initial survey of local general practitioners (GPs) and discussion with Oxfordshire local medical committee (LMC), we highlighted several key areas of discharge communication that were not satisfactory: recording of the grade and contact details of discharging doctor, medication changes, and clear documentation of outstanding follow-up. The local GPs were forthcoming with their criticism of current practice.

Sub-optimal content of elDDs could have been dependent on lack of formal training and education, but also influenced by the format of the eIDD proforma which previously lacked prompts for important information and failed to mandate completion of many sections. Our overall aims included tailoring discharge summary information to primary care needs; altering the electronic system to ensure required actions could be flagged for primary care physicians; and educating junior doctors of what actions are realistic to expect from GPs after patient discharge.

\section{Background}

Poor quality discharge summaries have been repeatedly demonstrated to lead to increased adverse events in patient care after discharge and need for re-hospitalization. Research has shown that there is poor information continuity after discharge from hospital.[1] However, a complete, accurate, and timely discharge summary can communicate important information to the GP, prevent adverse events, and reduce hospital readmission.[2]

Key elements of a good discharge summary include the identification of unresolved medical issues at discharge, results requiring follow-up, and the presence of an accurate discharge medication list. When these items are not listed, there can be negative impact on patient care and health outcomes. $[3,4]$ Indeed, several studies evaluating the completeness of discharge summaries have found that these elements are often lacking.[5,6] For example, Kripalani et al. showed in a systematic review that items such as the primary diagnosis, test results, discharge medications and follow-up plans were absent or incomplete in 14 to $38 \%$ of discharge letters.[6]

Anecdotally there appears to be little formal teaching about discharge summaries in the curricula of most medical schools,[7] yet a recent study has shown that simple, intensive educational sessions can lead to an improvement in discharge summaries and communication with primary care.[8]

\section{Baseline measurement}


We performed an initial survey of GPs in Oxfordshire (representatives suggested by the LMC), and from this we developed a list of "golden rules" for good discharge communication. They consisted of: providing an up-to-date medication list with changes clearly stated; including significant results (and MMSE where appropriate); stating diagnoses, follow-up arrangements (and avoiding phrases such as "GP please chase") and doctor's details; ensuring timely completion; and considering phoning the GP directly before discharge (figure 1).

According to these standards, we developed a proforma for audit data collection. With the help of foundation year 2 doctors (FY2s) working at Oxford GP surgeries, we performed a retrospective audit of discharge summaries delivered to six practices (randomly selected from a range of Oxfordshire practices employing FY2s) in April and May 2013 (332 discharge summaries). We recorded which information was missing and whether requested follow-up actions were completed in primary care.

This baseline data collection found that the grade or contact details of the discharging doctor was missing in 110 out of 332 elDDs (33\%). Medication changes were inadequately recorded in 226 elDDs (68\%), and in 73 cases (22\%), requested follow-up actions were not completed. Indeed, in 142 elDDs (44\%) there was no specific timeframe for such GP actions. Moreover, it took up to 51 days in one case (median one day) for an eIDD to reach the GP surgery, and up to 35 days (median zero days) for the GP to review the summary (figures 2 to 5 in results section).

See supplementary file: ds4952.pdf - "Figure 1: Tube Map of Golden Rules"

\section{Design}

All the involved parties (GPs, Oxford Deanery, Oxford University Hospitals (OUH) management) received the project proposal, including their expected role and asked for feedback. After obtaining their comments, we arranged a face-to-face multidisciplinary meeting presenting our survey results, promoting participants' networking and crucially, planned the intervention steps.

When analyzing the failures in the elDDs identified during our initial audit, it was clear that there was variation in doctors' performance and indeed no standardised way of setting out discharge information. Based on existing evidence for education improving quality of discharge summaries,[6] we decided that teaching would be a key intervention here.

Intervention design:

a. Foundation school teaching: An interactive workshop (90 min) stimulating FYDs to derive discharge communication "golden rules" using real discharge summary examples. We recruited experienced GPs to teach from a clinical school database for primary care educators. The foundation school incorporated this session into their official training curriculum (once a year). A shorter lecture-style teaching session (15 minute) was inserted into the Trust induction programme (every four months). A similar (but specialty-specific) session was suggested for induction to any hospital rotation

b. eIDD software change: The OUH Trust has implemented a new electronic patient

record system and we notified the technical support team of our activity. We suggested a drop-down menu of follow-up actions with prompts about realistic timing and appropriate use. Further, this system auto-populates the list of mediation changes since admission

c. E-learning module: OUH will host an e-learning session for those unable to attend live teaching. We aim to establish a medical student team for this work. If recruitment fails, a video record of the lecture-style teaching session will be used.

\section{Strategy}

PDSA cycle 1: Our initial "plan" phase came out of the audit phase and in our meetings with stakeholders. The "do" phase of this cycle involved two teaching sessions. These two teaching sessions were given at foundation year 1 and year 2 teaching as part of their scheduled curriculum coverage in March 2014. Our "study" step involved collecting feedback after this teaching. The feedback we received from foundation year one trainees was, on the whole, positive. The main criticism was that it would have been better placed at the beginning of the academic year when they were new to the wards.

For foundation year two doctors.feedback was less positive, and they felt it was unlikely to change their practice as they had been writing discharge summaries for over 18 months already. The second part of the "study" phase involved repeating our data collection about the quality of discharge summaries after these initial teaching sessions were delivered (the results of which can be seen in the results section below). Finally, for our "act" step we changed our project methods, recognising that we needed to change our teaching to earlier on in foundation year doctors' teaching schedule, and that other non-teaching interventions were needed to meet our aims.

PDSA cycle 2: The "plan" phase of cycle 2 followed on from the "act" phase of cycle 1. The feedback from our initial PDSA cycle led us to introduce a discharge documentation workshop within the foundation year 1 induction to the trust and 'Introduction to hospital medicine' week prior to starting on the wards. In this session, a local GP presented a personal perspective on the need for good discharge communication and one of the members of our quality improvement team presented the golden rules and the results of our project to date. After this "do" phase we again collected feedback as part of our "study" phase. This session received very positive feedback and the trainees were encouraging about how it would affect their discharge summary communication in their new jobs.

Furthermore, in this time period, the trust made changes to the electronic discharge format. It now includes an automated list of medication changes and a box for GP follow-up actions. 
The extension to the "study" phase will involve auditing the effects of these changes on the quality of discharge summaries. This is scheduled for April to May 2015. We felt it important to stick to the same time of year, as the junior doctors audited will be at the same point in training.

Prior to the start of the new academic year in August 2015, we hope to introduce an e-learning package as part of mandatory trust induction.

\section{Results}

The re-audit was performed in April and May 2014, one month following our first teaching interventions delivered to the OUH FYDs. One hundred and forty-two elDDs from four practices were analyzed in total against the same measures that had been used for our baseline data collection. We found $36 \%$ elDDs (51/142) did not have a documented grade of doctor, in $27 \%$ (38/142) requested follow-up did not take place, and in 50\% (71/142) there was no clear deadline for GP actions. Sixty-one percent (86/142) did not have clear documentation of medication changes. Using the Chi squared test, there was no statistically significant difference in the "quality" of elDDs before and after our first interventions (figure 2 to $5)$.

See supplementary file: ds4955.pdf - "Graphs comparing 'quality' of discharge summaries in 2013 and 2014."

\section{Lessons and limitations}

Expecting three junior doctors alone to drive this project at $\mathrm{OUH}$ seemed unrealistic. We found that the only way to succeed was to effectively engage stakeholders whose authority in the system opens doors towards implementation. Interestingly, many GPs felt initially threatened by this initiative, thinking it may lead to an increased burden of work transferred from secondary care. This tension only stressed the importance of our project in introducing missing standards into discharge communication.

Our initial audit included elDDs written by all grades of doctor. These were mainly done by FYDs or senior house officers (SHOs), but occasionally by registrars and even consultants. If we were to audit again, it may be useful to include only summaries written by FYDs so that we can ascertain whether our interventions are having an effect without diluting our sample. We should be looking at this at the same time each year, when the FYDs have had similar experience from induction.

In this project we had to involve many different stakeholders and consider their feedback. This contributed to the long timescale between our data collection and us implementing interventions. In the future, we could audit more often throughout the year, but the work required and the need for a large sample limits this.

There was variability in the way data was recorded from elDDs for the "medication changes" standard. Data collectors either used "yes"/ "no" to document if medication changes were clearly recorded, but some also recorded "N/A". Therefore in collating our data, we made the assumption that "yes" or "N/A" was adequate and met our standard, while "no" was deemed an inadequate documentation. This means our statistics for this measure may be less reliable and so for our next re-audit, we need to ensure clearer parameters for data recording.

Overall, we feel our interventions are sustainable. They are supported by the foundation school and a regular teaching session will remain both in mandatory Trust Induction and in foundation teaching for FYDs once a year.

We were fortunate that the OUH Trust was working on improving eIDD proforma structure in parallel with our project, enabling faster implementation of some recommended software changes. However, after this window of opportunity closes, further software changes may be more challenging to instigate.

\section{Conclusion}

Discharge communication is an essential component of transition from secondary to primary care. Our study identifies sub-optimal discharge communication at the OUH Trust, which is likely to be reflected in other trusts. In particular, the areas of concern include medication reconciliation and follow-up plans.

If health systems are to delegate discharge summaries to the less experienced team members, they should ensure clear guidance and standards, relevant training, and routine audit. As a result, we initiated teaching for FYDs on this subject. We have not yet demonstrated any improvement in the quality of elDDs following our first teaching intervention. It is likely that a combination of teaching, IT support, and greater accountability will ensure that harm is reduced as patients transition from secondary to primary care.

\section{References}

1. Walraven C, Taljaard M, Bell CM, Etchells E, Stiell IG, Zarnke K, Forster AJ. A prospective cohort study found that provider and information continuity was low after patient discharge from hospital. J Clin Epidemiol 2010;63:1000-10.

2. Walraven C, Seth R. Effect of Discharge Summary Availability During Post-discharge Visits on Hospital Readmission. J Gen Intern Med 2002;17:186-19.

3. Moore C, McGinn T, Halm E. Tying up loose ends: discharging patients with unre-solved medical issues. Arch Intern Med 2007;167:1305-11.

4. Perren A, Previsdomini M, Cerutti B, Soldini D, Donghi D, Marone C. Omitted and unjustified medications in the discharge summary. Qual Saf Health Care 2009;18:205-8.

5. Walraven C, Weinberg A. Quality of A Discharge Summary System. CMAJ 1995;152:1437-42.

6. Kripalani S, LeFevre F, Phillips CO, Williams MV, Basaviah $P$, Baker DW. Deficits in communication and information transfer between hospital-based and primary care physicians: implications for patient safety and continuity of care. JAMA 2007;297:831-41. 
7. Frain JP, Frain AE, Carr PH.Experience of medical senior house officers in preparing discharge summaries. BMJ 1996;312:350.

8. Shivji FS, Ramoutar DN, Bailey C, Hunter JB. Improving communication with primary care to ensure patient safety post-hospital discharge. Br J Hosp Med (Lond) 2015 Jan;76(1):46-9.

\section{Declaration of interests}

Nothing to declare.

\section{Acknowledgements}

QIP team members and authors: Doctors Matthew Hart, Stephen Hibbs, Ondrej Suchanek, Tania Young, and Laurence Leaver.

We would like to acknowledge Dr Lara Hibbs for her contribution to poster design.

Data contributors: Doctors Tom McWilliams, Rachel Carten, Sam Botchey, Emily Middleton, Marian Elfons, Deepa Panchalingam and Lucy Johnson.

\section{Ethical approval}

According to local trust policy, this project was deemed to constitute quality improvement not requiring formal ethics approval. It was not a study on human subjects and was approved by the local quality improvement lead. 